\title{
Effects of a parallel magnetic field on the ground-state magnetic properties of a two-dimensional electron gas
}

\author{
A. L. Subaşi and B. Tanatar \\ Department of Physics, Bilkent University, Bilkent, Ankara 06800, Turkey \\ (Received 23 August 2006; revised manuscript received 4 August 2008; published 3 October 2008)
}

\begin{abstract}
We study the magnetic behavior and in particular the spin magnetization of an interacting two-dimensional electron gas in an in-plane magnetic field. The ground-state energy of the system is constructed using the correlation energy based on the recent quantum Monte Carlo (QMC) simulations as a function of density, spin polarization, and applied magnetic field. The critical magnetic field to fully spin polarize the system is obtained as a function of the electron density. The spin polarization as a function of the applied field (less than the critical field) for various densities is calculated. When the QMC parametrization is employed, we find that the two-dimensional electron system undergoes a first-order phase transition to a ferromagnetic state in the regions $0<r_{s}<7$ and $20<r_{s}<25$, where $r_{s}$ is the usual density parameter. For $7<r_{s}<20$ our calculations indicate a second-order transition unlike approximate theories.
\end{abstract}

DOI: 10.1103/PhysRevB.78.155304

PACS number(s): 71.10.Ca, 72.25.Dc, 75.40.Cx, 73.20.At

\section{INTRODUCTION}

The ground-state properties of the two-dimensional electron-gas (2DEG) model are important not only for their technological implications but also from the point of view of many-body physics. ${ }^{1}$ In the last decade there has been a huge amount of activity on the transport and thermodynamic properties of low-density 2DEG systems largely motivated by the observed metal-insulator transition. ${ }^{2}$ In particular, the spin susceptibility of a $2 \mathrm{DEG}$ is of interest and many experimental studies are reported ${ }^{3-10}$ on Si-metal-oxide-semiconductor field-effect transistor (MOSFET) and GaAs based twodimensional (2D) electron systems. Irrespective of the material details the spin susceptibility is found to be enhanced with decreasing carrier density. ${ }^{11}$

On the theoretical side, the ground-state energy of the 2DEG is most reliably assessed from quantum Monte Carlo (QMC) simulations. ${ }^{12,13}$ In particular, the recent simulations predict a paramagnetic to ferromagnetic transition before the eventual crystallization of electrons and provide an accurate correlation energy in parametrized form. This allows the calculation of other thermodynamic quantities of interest without resorting to perturbation-theory approaches. Experimental observation of spontaneous spin polarization of a 2DEG has been reported by Ghosh et al. ${ }^{14}$ and Winkler et al. ${ }^{15}$ Recent spectroscopic measurements on the spin polarization in dilute semimagnetic quantum wells also shed some light on the exchange-correlation effects in 2D electron systems. ${ }^{16}$

In a recent paper Zhang and Das Sarma ${ }^{17}$ challenged the interpretation of most spin-susceptibility measurements by studying the spin-polarization effects in a $2 D E G$ in the presence of an applied magnetic field. The paramagnetic to ferromagnetic transition in electron systems has long been of interest ${ }^{18,19}$ and the recent experiments have revived further theoretical activity ${ }^{17,20-23}$ including a study on Dirac fermions in graphene. ${ }^{24}$

Motivated by the recent experiments on 2DEG systems with an in-plane magnetic field and the associated measurements of thermodynamic quantities, in this paper we revisit the calculation of spin-polarization effects taking advantage of the recent QMC simulation results ${ }^{13}$ which provide an accurate correlation energy with density and spin-polarization dependence. As the QMC simulations are performed for a strictly $2 \mathrm{DEG}$ system at zero temperature, $T=0$, we consider a similar system ignoring the finite width of quantum well structure. Thus, coupling of the magnetic field to the orbital motion does not enter the picture. Because we do not include any valley degeneracy effects, our calculations should be more appropriate for GaAs based electron and hole systems. The effects of finite width and disorder, treated perturbatively, on the spin susceptibility of a 2DEG have recently been considered by De Palo et al. ${ }^{25}$ Comparing our results with those of previous perturbation-theory based calculations we find qualitative as well as quantitative differences.

The rest of this paper is organized as follows. In Sec. II we provide the ground-state energy expression as a function of electron density, spin-polarization parameter, and applied magnetic field and outline our calculation of the critical field at full spin polarization. In Sec. III we present our numerical results and compare them with other theoretical approaches. We conclude with a brief summary in Sec. IV.

\section{MODEL AND THEORY}

We consider a 2DEG with zero layer thickness in the presence of uniform positive charges to ensure charge neutrality. At $T=0$ the system is characterized by the following two dimensionless parameters. The Wigner-Seitz radius $r_{s}$ defined in terms of the density $n$ and the effective Bohr radius $a_{B}^{*}$ by $n=1 / \pi\left(a_{B}^{*} r_{s}\right)^{2}$, which is the average distance between the electrons in units of $a_{B}^{*}$ (Bohr radius includes the band mass of electrons and the background dielectric constant of the host semiconducting material). The spin polarization is the ratio of the number of excess spin-up electrons to the total number of electrons given by $\zeta=\left|n_{\uparrow}-n_{\downarrow}\right| / n$. In the former case the system is said to be unpolarized and one talks about a paramagnetic state, whereas in the latter case, the system is fully polarized and is called ferromagnetic. The ratio of the average interaction energy to the average kinetic 
energy is also equal to $r_{s}$; thus small $r_{s}$ values characterize high-density and weakly interacting systems and large $r_{s}$ values characterize low-density and strongly interacting systems.

The total energy per particle in the presence of an in-plane applied magnetic field $B$ can be written in terms of the variables $r_{s}, \zeta$, and $B$ as

$$
E\left(r_{s}, \zeta, B\right)=E_{k}\left(r_{s}, \zeta\right)+E_{x}\left(r_{s}, \zeta\right)+E_{c}\left(r_{s}, \zeta\right)+E_{Z}(\zeta, B)
$$

where $E_{k}\left(r_{s}, \zeta\right)=\left(1+\zeta^{2}\right) / r_{s}^{2}$ is the kinetic energy per particle and $E_{x}\left(r_{s}, \zeta\right)=-\left(4 \sqrt{2} x / 3 \pi r_{s}\right)\left[(1+\zeta)^{3 / 2}+(1-\zeta)^{3 / 2}\right]$ is the exchange energy in units of effective Rydberg (Ry). For the correlation energy $E_{c}$ we use two models. The first one is given by the following parametrized expression from QMC calculations of Attaccalite et al.: ${ }^{13}$

$$
E_{c}\left(r_{s}, \zeta\right)=\left(e^{-\beta r_{s}}-1\right) \epsilon_{x}^{(6)}\left(r_{s}, \zeta\right)+\alpha_{0}\left(r_{s}\right)+\alpha_{1}\left(r_{s}\right) \zeta^{2}+\alpha_{2}\left(r_{s}\right) \zeta^{4}
$$

where

$$
\epsilon_{x}^{(6)}\left(r_{s}, \zeta\right)=E_{x}\left(r_{s}, \zeta\right)-\left(1+\frac{3}{8} \zeta^{2}+\frac{3}{128} \zeta^{4}\right) E_{x}\left(r_{s}, 0\right),
$$

and

$$
\begin{aligned}
\alpha_{i}\left(r_{s}\right)= & A_{i}+\left(B_{i} r_{s}+C_{i} r_{s}^{2}+D_{i} r_{s}^{3}\right) \\
& \times \ln \left(1+\frac{1}{E_{i} r_{s}+F_{i} r_{s}^{3 / 2}+G_{i} r_{s}^{2}+H_{i} r_{s}^{3}}\right) .
\end{aligned}
$$

The constants $A_{i}, \ldots, H_{i}$ in the functions $\alpha_{i}(i=0,1,2)$ are given in tabulated form by Attaccalite et al. ${ }^{13}$ When an inplane magnetic field is applied to the 2DEG system, the interaction of the magnetic field with the spin of the electrons gives rise to Zeeman energy $E_{Z}(\zeta, B)=-\frac{g}{2} \mu_{B} \zeta B$ (per particle), where $g$ is the effective band $g$ factor and $\mu_{B}$ is the Bohr magneton.

In the absence of an external magnetic field the recent QMC simulations predict spontaneous transition from a paramagnetic state to a ferromagnetic state around $r_{s} \approx 25.5$. Unlike the situation ${ }^{26}$ in three dimensions, to the accuracy of simulation results there is no partially polarized phase for the entire range of densities. However, when an external magnetic field is applied it becomes possible to polarize the system partially, and as the magnetic-field strength is further increased, the system becomes fully polarized at a critical value of the magnetic field. This critical or polarizing field can easily be found for a noninteracting system whose energy is simply given by $E_{0}\left(r_{s}, \zeta, B\right)=\left(1+\zeta^{2}\right) / r_{s}^{2}-\left(g \mu_{B} / 2\right) B \zeta$. The total energy for a noninteracting system with respect to the spin polarization $\zeta$ is a parabola, the minimum of which for small $B\left(B<B_{c 0}\right)$ occurs at $\zeta_{0}^{*}=g \mu_{B} r_{s}^{2} B / 4$. The critical field $B_{c 0}$ for the noninteracting case is found by setting $\zeta_{0}^{*}$ $=1$, yielding $B_{c 0}=4(\mathrm{Ry}) /\left(g \mu_{B} r_{s}^{2}\right)=2 \epsilon_{F} /\left(g \mu_{B}\right)$ in which $\epsilon_{F}$ is the Fermi energy of the unpolarized system. For higher fields the energy minimum is always at $\zeta_{0}^{*}=1$.

In the case of interacting particles, assuming the energy has a local minimum as a function of $\zeta$, we proceed in the same way to find the critical field. The optimum polarization $\zeta^{*}$ is again found by minimizing the total energy. The result-

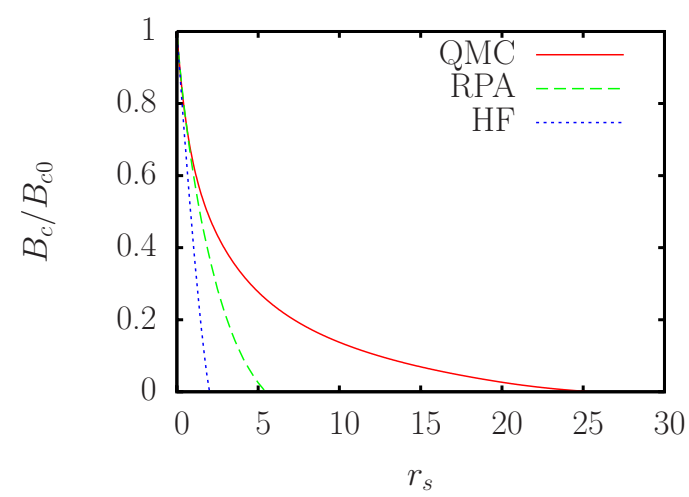

FIG. 1. (Color online) The critical field $B_{c}$ to fully polarize the 2DEG as a function of $r_{s}$ in various approximations, Hartree-Fock (dotted line), RPA (dashed line), and QMC correlation energy (solid line).

ing polarization $\zeta^{*}\left(r_{s}, B\right)$ that is now a function of $r_{s}$ and applied magnetic field, when set equal to unity, yields the critical field $B_{c}$ which can be written as

$$
\frac{B_{c}}{B_{0 c}}=1-\frac{2}{\pi} r_{s}+\frac{9 \sqrt{2}-8}{\pi}\left(e^{-\beta r_{s}}-1\right) r_{s}+\left(\alpha_{1}+2 \alpha_{2}\right) r_{s}^{2}
$$

In the above expression the first two terms on the right-hand side give the Hartree-Fock approximation (HFA) and the remaining terms follow from the parametrized form of the correlation energy $E_{c}$ from the QMC simulation.

The above calculation assumes that the minimum of total energy at the critical field $B_{c}$ occurs at $\zeta^{*}=1$. However, this is not always the case as a number of previous works based on the random-phase approximation (RPA) have already shown. ${ }^{17,18,22}$ If the minimum occurs at $\zeta^{*}=1$ the above formulas are valid and spin polarization approaches unity continuously yielding a second-order transition to the fully polarized state. As will be discussed in detail later, for some values of $r_{s}$ the form of the energy curve is fundamentally different from that of the noninteracting case. At the critical field $B_{c}$, the total energy as a function of polarization has two minima. One of them is at $\zeta^{*}=1$ and the other one is at 0 $<\zeta^{*}<1$. Since just beyond the critical field the global minimum occurs at $\zeta^{*}=1$, there is a discrete jump in the spin polarization and the transition is first order.

\section{RESULTS AND DISCUSSION}

We now present our results based on the above constructed ground-state energy of a $2 \mathrm{DEG}$ with an in-plane magnetic field. We have calculated the minimum of the ground-state energy with respect to spin polarization for various values of $r_{s}$ and $B$. The search for the critical field employed here is purely numerical and is an incremental search. The magnetic field is increased until the minimum of the energy occurs at $\zeta=1$.

The critical field $B_{c}$ that fully polarizes the electron gas as a function of $r_{s}$ in various theoretical models is plotted in Fig. 1. At points above each curve in the $r_{s}-B$ plane the 

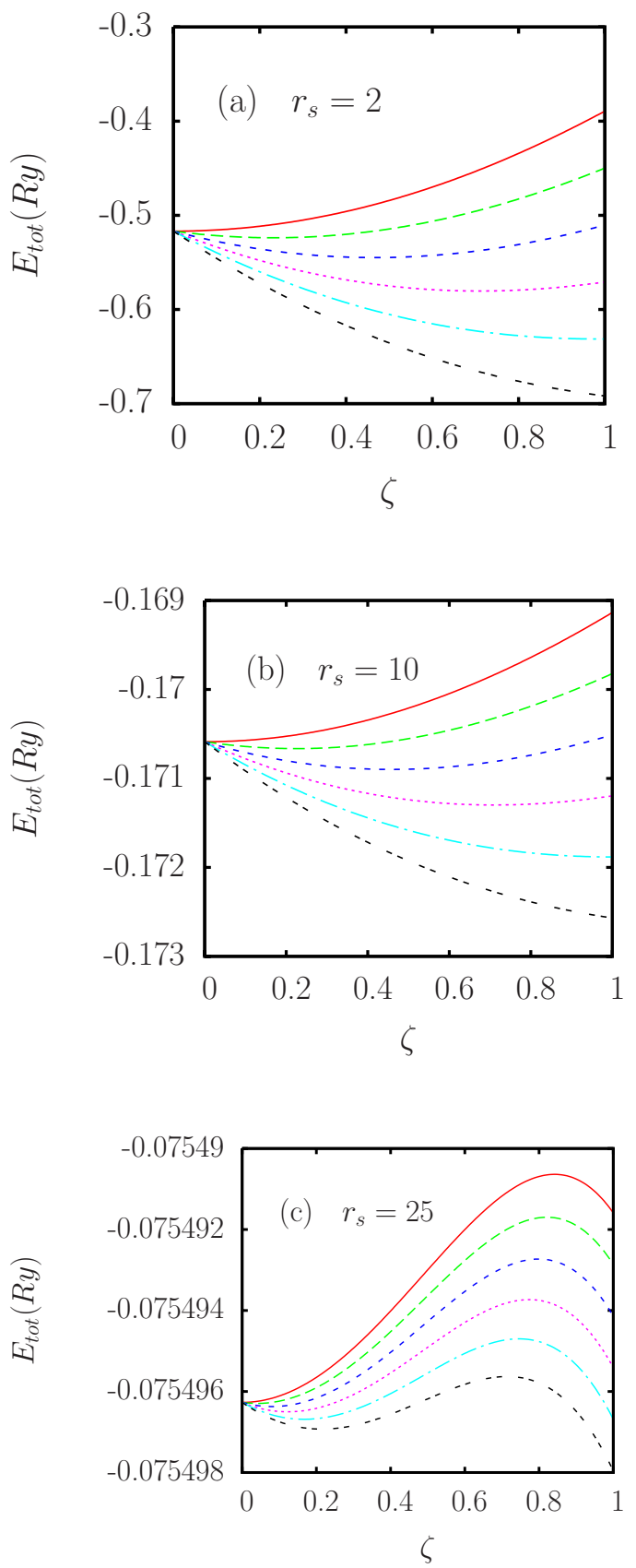

FIG. 2. (Color online) Ground-state energy as a function of spin polarization $\zeta$ at $r_{s}=2,10$, and 25 for various applied magnetic-field values (from top to bottom, $B=0,0.25 B_{c}, 0.5 B_{c}, 0.75 B_{c}, B_{c}$, and $\left.1.25 B_{c}\right)$.

system is fully polarized. The critical field vanishes around $r_{s}^{*} \sim 25.5$ when the QMC correlation energy of Attaccalite et al. ${ }^{13}$ is used, at which density of the system undergoes a spontaneous transition to the ferromagnetic state. Also given in the same figure are the corresponding curves for the HFA and RPA which show quantitatively different behavior from the present results including correlation effects.

The total-energy curves at increasing magnetic field as a function of the spin polarization for the two models of correlation energy at three representative values of $r_{s}$ are shown in Fig. 2. In this figure, we display the results using QMC correlation energy of Attaccalite et al. ${ }^{13}$ At zero field the

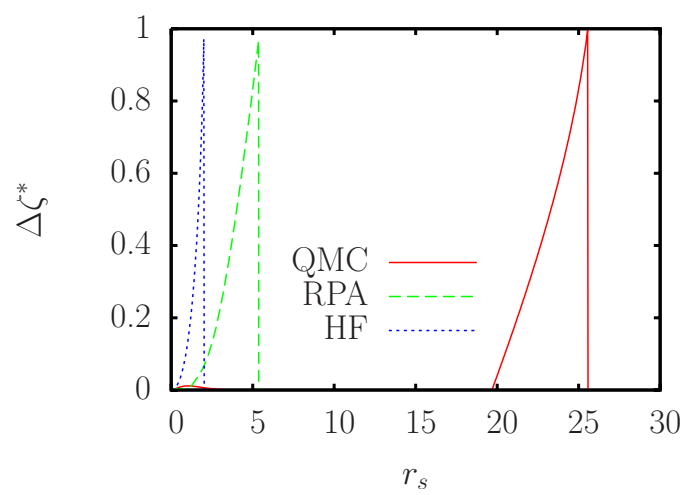

FIG. 3. (Color online) The discontinuous jump in spin polarization $\Delta \zeta^{*}$ at $B_{c}$ as a function of $r_{s}$. Dotted, dashed, and solid lines represent HFA, RPA, and QMC correlation energy, respectively.

minimum of the total energy is at $\zeta=0$ for $r_{s} \lesssim 25.5$. As the magnetic field is increased the minimum shifts to nonzero values of $\zeta$. For instance, at $r_{s}=2$ and $r_{s}=25$ the total energy has two minima when the field reaches the critical value $B_{c}$ at the corresponding density (for $r_{s}=2$ the local minimum and the minimum at $\zeta=1$ are very close and not visible on this scale). Above $B_{c}$ the energy has one minimum at the end point $\zeta=1$; there is an abrupt change in $\zeta$ at $B_{c}$. For $r_{s}=10$, on the other hand, we find that the local minimum moves to the right as the field increases but continuously goes to $\zeta=1$ at $B_{c}$.

The jump in the spin polarization at the critical field $B_{c}$ denoted by $\Delta \zeta^{*}$ is shown in Fig. 3. For the QMC correlation energy in the ranges $0<r_{s}<7$ and $20<r_{s}<25$ we find that there is a discrete jump in polarization which is equal to the distance between the two minima of energy. The transition to the polarized state is first order when $\Delta \zeta^{*} \neq 0$. Such a phase transition is known as Bloch ferromagnetism. For intermediate values of $r_{s}$ we find that the polarization becomes unity continuously as the magnetic field is increased. In this region the phase transition to the ferromagnetic state appears to be of Stoner type (i.e., second order). In contrast, approximate theories such as HFA and RPA yield a finite $\Delta \zeta^{*}$ in the $r_{s}$ regions of their applicability. The qualitatively different behavior found for $7<r_{s}<20$ implying a continuous phase transition to the ferromagnetic state is a direct result of our use of the parametrized QMC correlation energy. It is known that the energy differences between the polarized states are diminishingly small. Thus, the results of our calculations are limited by the accuracy of the parametrized QMC expression. The small jump in polarization for $0<r_{s}<7$ is intriguing. To further check the robustness of this prediction we have used the correlation energy expression recently proposed by Chesi and Giuliani. ${ }^{27}$ In this work differences from QMC results in spin polarized energies are reported. Although the Gell-Mann-Bruckner-type calculation of Chesi and Giuliani ${ }^{27}$ is only valid for $r_{s} \rightarrow 0$, we have found that a small nonzero $\Delta \zeta^{*}$ up to $r_{s} \approx 1$. Thus, it appears that for small $r_{s}$ there is a weak first-order transition to the ferromagnetic state.

The spin polarization $\zeta^{*}$ that minimizes the ground-state 

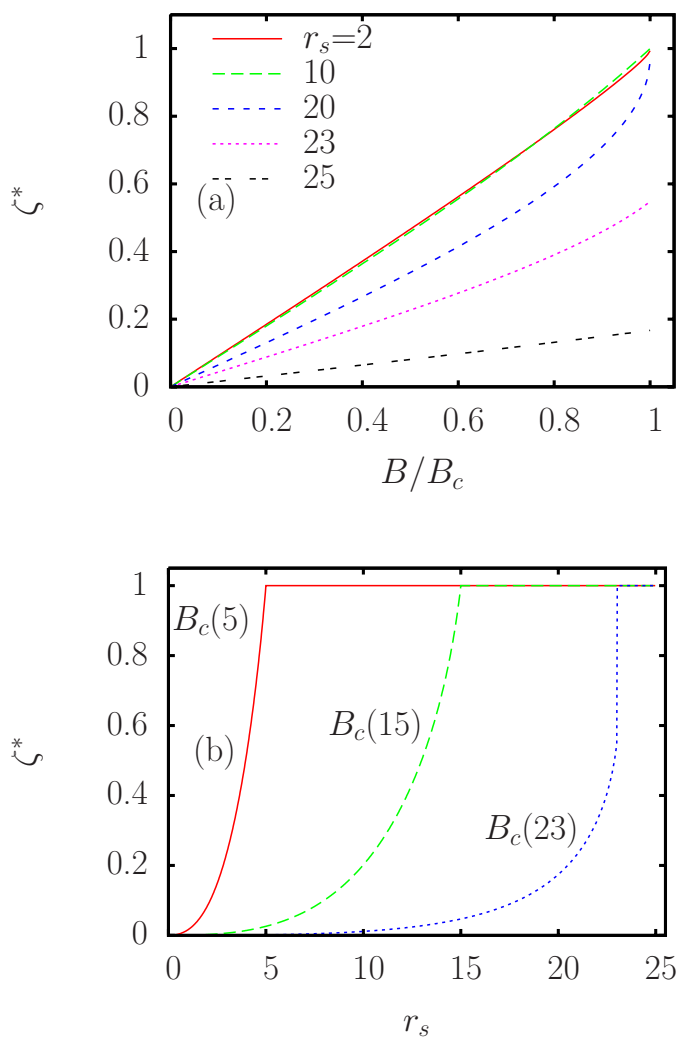

FIG. 4. (Color online) Spin polarization $\zeta^{*}$ (the value of $\zeta$ which minimizes the ground-state energy at a given magnetic field) as a function of (a) the $B$ field for several $r_{s}$ values and (b) as a function of $r_{s}$ for several $B$-field values. Solid, dashed, and dotted lines indicate $B=B_{c}\left(r_{s}=5\right), B=B_{c}\left(r_{s}=10\right)$, and $B=B_{c}\left(r_{s}=20\right)$, respectively.

energy is shown in Fig. 4 as a function of $B$ field at fixed density and as a function of $r_{s}$ at constant $B$. For those values of $r_{s}$ shown in Fig. 4(a) there is a jump before $\zeta^{*}$ becomes unity at $B_{c}$ consistent with the results presented in Fig. 3 where $\Delta \zeta^{*}>0$. As $\Delta \zeta^{*}=0$ for $7<r_{s}<20$, we find that $\zeta^{*}(B)$ curves approach unity smoothly in this region. In Fig. 4(b) we show $\zeta^{*}$ as a function of $r_{s}$ at the constant magnetic-field values of $B_{c}\left(r_{s}=5\right), B_{c}\left(r_{s}=10\right)$, and $B_{c}\left(r_{s}=10\right)$. Thus the plotted curves exhibit the onset of full spin polarization as the density is decreased. Note also that nonzero values of $\Delta \zeta^{*}$ consistent with those shown in Fig. 3 are clearly visible.

Another quantity of interest which can be accessed experimentally is the magnetic susceptibility of the system defined as $\chi=n\left(g \mu_{B} / 2\right) \partial \zeta^{*} / \partial B$. It is common practice to look at the susceptibility normalized by its value $\chi_{0}$ for the noninteracting system (Pauli susceptibility), i.e., $\chi_{0}=n \mu_{B}^{2} / \epsilon_{F}$, so that the ratio $\chi / \chi_{0}=\left(g \epsilon_{F} / 2 \mu_{B}\right) \partial \zeta^{*} / \partial B$ is formed. Using the analytic expressions for the various terms of the ground-state energy, we find

$$
\frac{\chi}{\chi_{0}}=\frac{2}{r_{s}^{2}}\left[\frac{2}{r_{s}^{2}}-\frac{\sqrt{2}}{\pi r_{s}}\left[\left(1+\zeta^{*}\right)^{-1 / 2}+\left(1-\zeta^{*}\right)^{-1 / 2}\right]+\left.\frac{\partial^{2} E_{c}}{\partial \zeta^{2}}\right|_{\zeta^{*}}\right]^{-1} .
$$

Thus, once having obtained $\zeta^{*}$ numerically, we can readily calculate the normalized susceptibility.
The zero-field (linear) susceptibility has been calculated by Attaccalite et al. ${ }^{13}$ On the other hand, the spin susceptibility at finite $B$ (nonlinear susceptibility) should be quantitatively different from calculations based on perturbation theory (HFA, RPA). The strong dependence on $r_{s}$ at finite fields is already evident in the magnetization curves of $\zeta^{*}(B)$ shown in Fig. 4.

Zhang and Das Sarma ${ }^{17}$ pointed out that spin susceptibility measured by magnetoresistance experiments ${ }^{3-7,9}$ through the polarization field $B_{c}$ does not coincide either with the linear or the nonlinear spin susceptibility, casting some doubt on the interpretation of experiments. The spin susceptibility is extracted from the measured $B_{c}$ that is related to a model dependence of $\zeta^{*}(B)$ which is typically linear. If the QMC parametrization gives a correct description with $\Delta \zeta^{*}=0$ for a range of $r_{s}$ values, the assumption about the slope of $\zeta^{*}$ vs $B$ appears to be reasonable. This coincides with the region 0 $<r_{s}<20$. In fact, since most experiments ${ }^{7-10}$ are performed at $r_{s} \lesssim 10$ experimental procedure seems to be valid. However, when $\Delta \zeta^{*}>0$ as in the case large $r_{s}$ region, then the experimental error would be considerable.

We also mention the recently reported thermodynamic measurements by Kravchenko et al. ${ }^{28}$ of the magnetization in a 2DEG. Spin susceptibility obtained by such measurements should provide an independent check of the same quantity from transport measurements. A related quantity, thermodynamic compressibility, also yields interesting features when the $2 \mathrm{DEG}$ is subjected to an in-plane magnetic field as we have discussed elsewhere. ${ }^{29}$

\section{SUMMARY AND CONCLUDING REMARKS}

We have considered the effect of in-plane magnetic field on the ground-state energy and magnetic properties of a 2DEG for a wide range of densities. To this purpose we have used the recently available QMC simulation based correlation energy as a function of $r_{s}$ and $\zeta$. Thus, our calculations should provide quantitatively more accurate results compared to the previously employed approximate methods. Interestingly, from the QMC correlation energy calculations we find that under an externally applied magnetic field the $2 \mathrm{D}$ electron system undergoes a first-order phase transition to a ferromagnetic state in the range $0<r_{s}<7$ and $20<r_{s}<25$. That is, as the magnetic field is increased from just below $B_{c}$ to above $B_{c}$, the polarization minimizing the total energy $\zeta^{*}$ jumps from a finite value to unity abruptly. On the other hand, in the range $7<r_{s}<20, \zeta^{*}$ reaches unity continuously which suggests a second-order phase transition. These findings are in qualitative difference with the predictions of HFA and RPA based calculations ${ }^{17}$ which yield a first-order phase transition to the ferromagnetic state in the whole range of densities corresponding to $0<r_{s} \lessgtr 5.5$.

There are several directions with which our calculations can be extended. To make better contact with experiments it would be useful to take the finite quantum well width effects into account. This would require a reliable calculation of the exchange and correlation energies as a function of $r_{s}, \zeta$, and parameters describing the finite width of electron layer, 
which presently are not available from QMC simulations. Furthermore, disorder effects are also likely to significantly affect the spin susceptibility and compressibility. It would be interesting to include the disorder effects in a realistic way when a direct comparison to the experiments is made.

\section{ACKNOWLEDGMENTS}

This work was supported by TUBITAK (Grant No. 106T052) and TUBA. We acknowledge useful discussions with M. Ö. Oktel, R. Asgari, and G. Senatore.
${ }^{1}$ T. Ando, A. B. Fowler, and F. Stern, Rev. Mod. Phys. 54, 437 (1982); G. F. Giuliani and G. Vignale, Quantum Theory of the Electron Liquid (Cambridge University Press, Cambridge, England, 2005).

${ }^{2}$ E. Abrahams, S. V. Kravchenko, and M. P. Sarachik, Rev. Mod. Phys. 73, 251 (2001); S. V. Kravchenko and M. P. Sarachik, Rep. Prog. Phys. 67, 1 (2004).

${ }^{3}$ T. Okamoto, K. Hosoya, S. Kawaji, and A. Yagi, Phys. Rev. Lett. 82, 3875 (1999).

${ }^{4}$ A. A. Shashkin, S. V. Kravchenko, V. T. Dolgopolov, and T. M. Klapwijk, Phys. Rev. Lett. 87, 086801 (2001).

${ }^{5}$ V. M. Pudalov, M. E. Gershenson, H. Kojima, N. Butch, E. M. Dizhur, G. Brunthaler, A. Prinz, and G. Bauer, Phys. Rev. Lett. 88, 196404 (2002).

${ }^{6}$ S. A. Vitkalov, M. P. Sarachik, and T. M. Klapwijk, Phys. Rev. B 65, 201106(R) (2002).

${ }^{7}$ E. Tutuc, S. Melinte, and M. Shayegan, Phys. Rev. Lett. 88, 036805 (2002); E. Tutuc, S. Melinte, E. P. De Poortere, M. Shayegan, and R. Winkler, Phys. Rev. B 67, 241309(R) (2003).

${ }^{8}$ J. Zhu, H. L. Stormer, L. N. Pfeiffer, K. W. Baldwin, and K. W. West, Phys. Rev. Lett. 90, 056805 (2003).

${ }^{9}$ K. Vakili, Y. P. Shkolnikov, E. Tutuc, E. P. De Poortere, and M. Shayegan, Phys. Rev. Lett. 92, 226401 (2004); Y. P. Shkolnikov, K. Vakili, E. P. De Poortere, and M. Shayegan, ibid. 92, 246804 (2004).

${ }^{10}$ Y.-W. Tan, J. Zhu, H. L. Stormer, L. N. Pfeiffer, K. W. Baldwin, and K. W. West, Phys. Rev. B 73, 045334 (2006).

${ }^{11}$ H. Boukari, F. Perez, D. Ferrand, P. Kossacki, B. Jusserand, and J. Cibert, Phys. Rev. B 73, 115320 (2006).

${ }^{12}$ B. Tanatar and D. M. Ceperley, Phys. Rev. B 39, 5005 (1989).

${ }^{13}$ C. Attaccalite, S. Moroni, P. Gori-Giorgi, and G. B. Bachelet, Phys. Rev. Lett. 88, 256601 (2002).

${ }^{14}$ A. Ghosh, C. J. B. Ford, M. Pepper, H. E. Beere, and D. A.
Ritchie, Phys. Rev. Lett. 92, 116601 (2004).

${ }^{15}$ R. Winkler, E. Tutuc, S. J. Papadakis, S. Melinte, M. Shayegan, D. Wasserman, and S. A. Lyon, Phys. Rev. B 72, 195321 (2005).

${ }^{16}$ C. Aku-Leh, F. Perez, B. Jusserand, D. Richards, W. Pacuski, P. Kossacki, M. Menant, and G. Karczewski, Phys. Rev. B 76, 155416 (2007).

${ }^{17}$ Y. Zhang and S. Das Sarma, Phys. Rev. Lett. 96, 196602 (2006).

${ }^{18}$ A. K. Rajagopal and J. C. Kimball, Phys. Rev. B 15, 2819 (1977); A. K. Rajagopal, S. P. Singhal, M. Banerjee, and J. C. Kimball, ibid. 17, 2262 (1978).

${ }^{19}$ R. Asgari, B. Davoudi, and M. P. Tosi, Solid State Commun. 131, 301 (2004); B. Davoudi and M. P. Tosi, Physica B (Amsterdam) 322, 124 (2002); R. Asgari, A. Esmailian, and B. Tanatar, Solid State Commun. 141, 595 (2007).

${ }^{20}$ F. Perrot and M. W. C. Dharma-wardana, Phys. Rev. Lett. 87, 206404 (2001).

${ }^{21}$ Y. Zhang and S. Das Sarma, Phys. Rev. B 72, 115317 (2005); 72, 125303 (2005).

${ }^{22}$ J. Terada and T. Ando, Physica E (Amsterdam) 34, 367 (2006).

${ }^{23}$ L. O. Juri and P. I. Tamborenea, Eur. Phys. J. B 45, 9 (2005).

${ }^{24}$ M. W. C. Dharma-wardana, Solid State Commun. 140, 4 (2006).

${ }^{25}$ S. De Palo, M. Botti, S. Moroni, and G. Senatore, Phys. Rev. Lett. 94, 226405 (2005).

${ }^{26}$ F. H. Zong, C. Lin, and D. M. Ceperley, Phys. Rev. E 66, 036703 (2002).

${ }^{27}$ S. Chesi and G. F. Giuliani, Phys. Rev. B 75, 153306 (2007).

${ }^{28}$ S. V. Kravchenko, A. A. Shashkin, S. Anissimova, A. Venkatesan, M. R. Sakr, V. T. Dolgopolov, and T. M. Klapwijk, Ann. Phys. (N.Y.) 321, 1588 (2006).

${ }^{29}$ A. L. Subasi and B. Tanatar, Solid State Commun. 144, 521 (2007). 